Published in: Lecture Notes in Computer Science 2415, 939-944 (2002).

\title{
Asymptotic Level Density of the Elastic Net Self-Organizing Feature Map
}

\author{
Jens Christian Claussen and Heinz Georg Schuster \\ Institut für Theoretische Physik und Astrophysik \\ Leibnizstr. 15, 24098 Christian-Albrechts-Universität zu Kiel, Germany \\ http://www.theo-physik.uni-kiel.de/ claussen/
}

\begin{abstract}
Whileas the Kohonen Self Organizing Map shows an asymptotic level density following a power law with a magnification exponent $2 / 3$, it would be desired to have an exponent 1 in order to provide optimal mapping in the sense of information theory. In this paper, we study analytically and numerically the magnification behaviour of the Elastic Net algorithm as a model for self-organizing feature maps. In contrast to the Kohonen map the Elastic Net shows no power law, but for onedimensional maps nevertheless the density follows an universal magnification law, i.e. depends on the local stimulus density only and is independent on position and decouples from the stimulus density at other positions.
\end{abstract}

Self Organizing Feature Maps map an input space, such as the retina or skin receptor fields, into a neural layer by feedforward structures with lateral inhibition. Biological maps show as defining properties topology preservation, error tolerance, plasticity (the ability of adaptation to changes in input space), and self-organized formation by a local process, since the global structure cannot be coded genetically. The self-organizing feature map algorithm proposed by Kohonen [1] has become a successful model for topology preserving primary sensory processing in the cortex 2], and an useful tool in technical applications [3.

The Kohonen algorithm for Self Organizing Feature Maps is defined as follows: Every stimulus $\mathbf{v}$ of an euclidian input space $V$ is mapped to the neuron with the position $\mathbf{s}$ in the neural layer $R$ with the highest neural activity, given by the condition

$$
\left|\mathbf{w}_{\mathbf{s}}-\mathbf{v}\right|=\min _{\mathbf{r} \in R}\left|\mathbf{w}_{\mathbf{r}}-\mathbf{v}\right|
$$

where |.| denotes the euclidian distance in input space. In the Kohonen model the learning rule for each synaptic weight vector $\mathbf{w}_{\mathbf{r}}$ is given by

$$
\mathbf{w}_{\mathbf{r}}^{\text {new }}=\mathbf{w}_{\mathbf{r}}^{\text {old }}+\eta \cdot g_{\mathbf{r s}} \cdot\left(\mathbf{v}-\mathbf{w}_{\mathbf{r}}^{\text {old }}\right)
$$

with $g_{\mathbf{r s}}$ as a gaussian function of euclidian distance $|\mathbf{r}-\mathbf{s}|$ in the neural layer. The function $g_{\mathbf{r s}}$ describes the topology in the neural layer. The parameter $\eta$ determines the speed of learning and can be adjusted during the learning process. Topology preservation is enforced by the common update of all weight vectors whose neuron $\mathbf{r}$ is adjacent to the center of excitation $\mathbf{s}$. 


\section{The Elastic Net Feature Map}

The Elastic Net 4 was proposed for solving optimization problems like the famous Travelling Salesman Problem. Here we apply this concept to feature maps. The Elastic Net is defined as a gradient descent in the energy landscape

$$
E=-\sigma^{2} \sum_{\mu} \ln \sum_{r} e^{-\left(\mathbf{v}^{\mu}-\mathbf{w}_{r}\right)^{2} / 2 \sigma^{2}}+\frac{\tilde{\kappa}}{2} \sum_{r}\left|\mathbf{w}_{r+1}-\mathbf{w}_{r}\right|^{2}
$$

with the input vectors denoted by $\mathbf{v}^{\mu}$. Here $r$ is the index of the neurons in an one-dimensional array (for the TSP: with periodic boundary conditions), and $\mathbf{w}_{r}$ is the synaptic weight vector of that neuron. For $\sigma \rightarrow 0$ (3) becomes

$$
\lim _{\sigma \rightarrow 0} E=\frac{1}{2} \sum_{\mu}\left(\mathbf{v}^{\mu}-\mathbf{w}_{s\left(\mathbf{v}^{\mu}\right)}\right)^{2}+\frac{\tilde{\kappa}}{2} \sum_{r}\left|\mathbf{w}_{r+1}-\mathbf{w}_{r}\right|^{2} .
$$

Here $s\left(\mathbf{v}^{\mu}\right)$ denotes the neuron with the smallest distance to the stimulus, the winning neuron, which is assumed to be nondegenerate. A gradient descent in the first term (which can be interpreted as an entropy term [5]) leads for sufficiently small $\sigma$ to the condensation of (at least) one weight vector to each input vector, if the input space is discrete. The second term is the potential energy of an elastic string between the weight vectors, and gradient descent in this term leads to a minimization of the (squared!) distances between the weight vectors.

Depending on parameter adjustment [6, 7] a gradient descent in $E$ can provide near-optimal solutions to the TSP within polynomial processing time [8], similar as the Kohonen algorithm [3]. We remark that in the Travelling Salesman application (if the numbers of neurons and cities are chosen to be equal) both the Elastic Net and the Kohonen algorithm share the same zero [3] and first 9] order terms and are therefore related for the final state of convergence, although their initial ordering process is different.

The update rule of the Elastic Net Algorithm is the gradient descent in (3):

$$
\frac{1}{\eta} \delta \mathbf{w}_{\mathbf{r}}=\sum_{\mu}\left(\mathbf{v}^{\mu}-\mathbf{w}_{\mathbf{r}}\right) \frac{e^{-\left(\mathbf{v}^{\mu}-\mathbf{w}_{\mathbf{r}}\right)^{2} / 2 \sigma^{2}}}{\int d \mathbf{r}^{\prime} e^{-\left(\mathbf{v}^{\mu}-\mathbf{w}_{\mathbf{r}^{\prime}}\right)^{2} / 2 \sigma^{2}}}+\tilde{\kappa} \Delta \mathbf{w}_{\mathbf{r}},
$$

where $\triangle w_{r}=w_{r-1}-2 w_{r}+w_{r+1}$ denotes the discrete Laplacian.

If we apply this concept to feature maps, we have to replace the sum over all input vectors by an integral over $\int p(\mathbf{v}) \mathrm{d} \mathbf{v}$, i.e. a probability density. If we interpret (5) as a neural feature mapping algorithm, it is a pattern parallel learning rule, or batch update rule, where contributions of all patterns are summed up to one update term. In the brain, hovever, patterns are presented serially in a stochastic sequence. Therefore we generalize this algorithm to serial presentation:

$$
\frac{1}{\eta} \delta \mathbf{w}_{\mathbf{r}}=\left(\mathbf{v}-\mathbf{w}_{\mathbf{r}}\right) \frac{e^{-\left(\mathbf{v}-\mathbf{w}_{\mathbf{r}}\right)^{2} / 2 \sigma^{2}}}{\int d \mathbf{r}^{\prime} e^{-\left(\mathbf{v}-\mathbf{w}_{\mathbf{r}^{\prime}}\right)^{2} / 2 \sigma^{2}}}+\kappa \Delta \mathbf{w}_{\mathbf{r}} .
$$


In Monte Carlo simulations of this model, one chooses input vectors $\mathbf{v}$ according to the probablility density function $p(\mathbf{v})$ and updates $\mathbf{w}_{\mathbf{r}}$ for every neuron $\mathbf{r}$ in the neural layer according to (6). The algorithm can be viewed as a stochastic approximation algorithm that converges if the conditions $\sum_{t=0}^{\infty} \eta^{2}(t)<\infty$ and $\sum_{t=0}^{\infty} \eta(t)=\infty$ for the time development of parameter $\eta$ are fulfilled [10] The simultaneous adjustment of $\kappa$ and $\sigma$ has been discussed in [6, 7] for the special case of the TSP optimization problem. For the TSP it appears necessary to adjust $\kappa / \sigma$ to a system-size-dependent value to avoid 'spike defects' for small $\kappa / \sigma$ and 'frozen bead defects' for large $\kappa / \sigma$ when annealing $\sigma \rightarrow 0$ [7. Both 'defects' are no defects in feature maps, the 'spike defects' can only occur for delta-peaked stimuli (cities) together with a dimension-reduction.

The aim in feature maps is different. Using the Kohonen algorithm, one tries to start with large-ranged interaction in the neural layer to avoid global topological defects. This is not directly possible for the Elastic Net, as its learning cooperation is restricted to next-neighbour. Only the strength of the elastic spring $\kappa$ can be initialized with a high value and decreased after global ordering. The parameter $\sigma$ is to be interpreted as a resolution length in feature space, e. g. the distance between two receptors in skin or retina. For selectivity of the winner-take-all mechanism, one would choose $\sigma$ smaller or alike the average or minimal distance between adjacent weight vectors.

\section{Asymptotic Density and the Magnification Fac- tor}

In this paper we consider the case of continuously distributed input spaces with same dimensionality as the neural layer, so there is no reduction of dimension.

The magnification factor is defined as the density of neurons $\mathbf{r}$ (i. e. the density of synaptic weight vectors $\mathbf{w}_{\mathbf{r}}$ ) per unit volume of input space, and therefore is given by the inverse Jacobian of the mapping from input space to neural layer: $M=|J|^{-1}=|\operatorname{det}(d \mathbf{w} / d \mathbf{r})|^{-1}$. (In the following we consider the case of noninverting mappings, where $J$ is positive.) The magnification factor is a property of the networks' response to a given probability density of stimuli $P(\mathbf{v})$. To evaluate $M$ in higher dimensions, one in general has to compute the equilibrium state of the whole network using global knowledge on $P(\mathbf{v})$.

For one-dimensional mappings (and possibly for special geometric cases in higher dimensions) the magnification factor may follow an universal magnification law, i.e. $M(\overline{\mathbf{w}}(\mathbf{r}))$ is a function only of the local probability density $P$ and independent of both location $\mathbf{r}$ in the neural layer and $\overline{\mathbf{w}}(\mathbf{r})$ in input space.

An optimal map from the view of information theory would reproduce the input probability exactly $\left(M \sim P(\mathbf{v})^{\rho}\right.$ with $\left.\rho=1\right)$, according to a power law with exponent 1 , equivalent to all neurons in the layer fire with same probability. An algorithm of maximizing mutual information has been given by Linsker 11.

For the classical Kohonen algorithm the magnification law (for one-dimensional mappings) is a power law $M(\overline{\mathbf{w}}(\mathbf{r})) \propto P(\overline{\mathbf{w}}(\mathbf{r}))^{\rho}$ with exponent $\rho=\frac{2}{3}$ [12]. For a discrete neural layer and especially for neighborhood kernels with different shape and range there are corrections to the magnification law [3, 13, 14]. 


\section{Magnification Exponent of the Elastic Net}

The necessary condition for the final state of algorithm (6) is that for all neurons $\mathbf{r}$ the expectation value of the learning step vanishes:

$$
\forall_{\mathbf{r} \in R} \quad 0=\int \mathrm{d} \mathbf{v} p(\mathbf{v}) \delta \mathbf{w}_{\mathbf{r}}(\mathbf{v}) .
$$

Since this expectation value is equal to the learning step of the pattern parallel rule (6), equation (7) is the stationary state condition for both serial and parallel updating. Inserting the learning rule (6) to condition (17), we obtain for the invariant density $\bar{w}_{r}$ in the one-dimensional case:

$$
0=\int\left(\left(v-\bar{w}_{r}\right) \frac{e^{-\left(v-\bar{w}_{r}\right)^{2} / 2 \sigma^{2}}}{\int d r^{\prime} e^{-\left(v-\bar{w}_{r^{\prime}}\right)^{2} / 2 \sigma^{2}}}+\kappa \Delta \bar{w}_{r}\right) P(v) d v .
$$

In the limit of a continuous neural layer for every stimulus $v$ there exists one unique center of excitation $s$ with $v=w_{s}$. Thus we can substitute integration over $d v$ by integration over $d s$. Using the Jacobian $J(s):=d \bar{w}(s) / d s$, we have

$$
0=\int\left((\bar{w}(s)-\bar{w}(r)) \frac{e^{-(\bar{w}(s)-\bar{w}(r))^{2} / 2 \sigma^{2}}}{\int d r^{\prime} e^{-\left(\bar{w}(s)-\bar{w}\left(r^{\prime}\right)\right)^{2} / 2 \sigma^{2}}}+\kappa \Delta \bar{w}(r)\right) P(\bar{w}(s)) J(s) d s .
$$

The second term becomes $\kappa \frac{\mathrm{d} J}{\mathrm{~d} r}$. The normalization integral is $\left(p:=s-r^{\prime}\right)$ :

$$
\int e^{-\left(\bar{w}(s)-\bar{w}\left(r^{\prime}\right)\right)^{2} / 2 \sigma^{2}} d r^{\prime}=\int e^{-p^{2} / 2(\sigma / J(s))^{2}} d p+o\left(\sigma^{3}\right)=\sqrt{2 \pi} \cdot \frac{\sigma}{J(s)}+o\left(\sigma^{3}\right) .
$$

For the following equations, we define the abbreviation $\bar{P}(r):=P(\bar{w}(r))$. Using parametric differentiation, substitution $\mathrm{d} s=\mathrm{d} w_{s} /\left(\mathrm{d} w_{s} / d s\right)=\mathrm{d} w_{s} / J(s)$, and saddlepoint expansion (method of steepest descent) for $\sigma \rightarrow 0$, the first integral becomes (after Simic [15]):

$$
\begin{aligned}
\frac{1}{\sqrt{2 \pi} \sigma} \cdot \int(\bar{w}(s)-\bar{w}(r)) e^{-(\bar{w}(s)-\bar{w}(r))^{2} / 2 \sigma^{2}} P(\bar{w}(s)) J(s)^{2} d s \\
=\frac{\sigma}{\sqrt{2 \pi}} \frac{1}{J(r)} \frac{d}{d r} \int e^{-(\bar{w}(s)-\bar{w}(r))^{2} / 2 \sigma^{2}} P(\bar{w}(s)) J(s)^{2} d s \\
=\frac{\sigma}{\sqrt{2 \pi}} \frac{1}{J(r)} \frac{d}{d r} \int e^{-(\bar{w}(s)-\bar{w}(r))^{2} / 2 \sigma^{2}} P(\bar{w}(s)) J(w(s)) d w(s) \\
=\sigma^{2} \frac{1}{J(r)} \frac{d}{d r}(\bar{P}(r) J(r))+o\left(\sigma^{4}\right)=\sigma^{2}\left(\frac{d \bar{P}}{d r}+\frac{\bar{P}}{J} \frac{d J}{d r}\right)+o\left(\sigma^{4}\right) .
\end{aligned}
$$

Neglecting higher orders of $\sigma$, we obtain

$$
0=\frac{\sigma^{2}}{J(r)} \cdot \frac{d}{d r}\left(\bar{P} J+\kappa \frac{d J}{d r}\right)
$$

This is a first-order nonlinear differential equation for $J(r)$ to a given input density $P(\bar{r})$. However, this can be expressed explicitly only if (additional to $P(v)$ ) the complete equilibrium state $\bar{w}(r)$ is known, and then one obtains $J(r)$ directly by evaluating the first derivative. Thus the differential equation (9) gives further insight only if $J(r)$ follows an universal scaling law without explicit dependence on the location $r$, that is, $J$ is a function of $\bar{P}$ only. 
The ansatz $J(r)=J(\bar{P}(r))$ leads for all $r$, where $d \bar{P} / d r \neq 0$, to the differential equation for the invariant state of the one-dimensional Elastic Net Algorithm

$$
\frac{d J}{d \bar{P}}=-\frac{J}{\bar{P}} \cdot\left(1+\frac{\kappa}{\sigma^{2}} \frac{J}{\bar{P}}\right)^{-1}
$$

The first derivative depends only on $J / \bar{P}$. The gradient field of (10) has two regimes: For $\kappa / \sigma^{2} \rightarrow 0$ ('soft string tension') $d J / d \bar{P}=-J / \bar{P}$, therefore $M=$ $J^{-1} \sim P(v)^{1}$. The magnification exponent is asymptotically 1 and cortical representation is near to the optimum given by information theory. For $\kappa / \sigma^{2} \rightarrow \infty$ ('hard string tension') $d J / d \bar{P} \rightarrow 0$, therefore $M=J^{-1}$ has a constant value. Here all adaptation to the stimuli vanishes, equivalent to a magnification exponent of zero.

Substituting $X:=\ln P, Y:=-\ln J$ and $Z:=X+Y$, (10) can be solved exactly (see Fig. 1)

$$
\ln M=\frac{1}{2}\left(\ln (P M)+\ln \left(1+\frac{1}{2} \frac{\kappa}{\sigma^{2}} \frac{1}{P M}\right)\right)+\text { const. }
$$

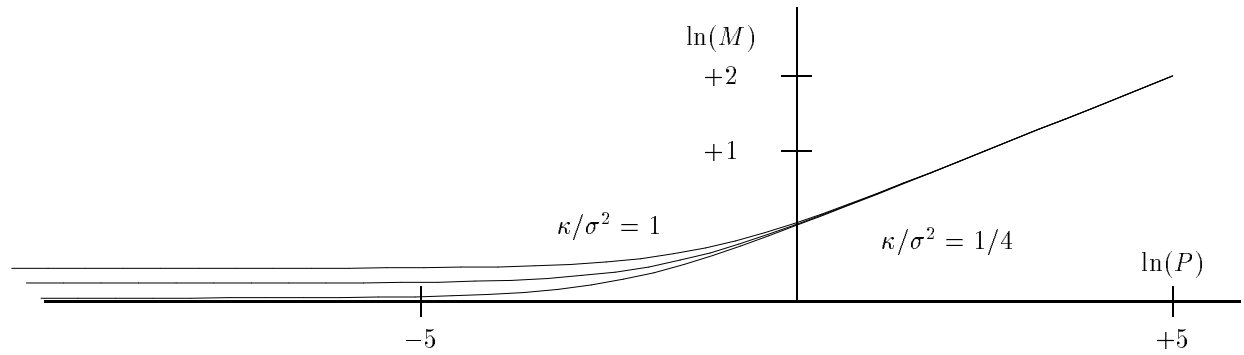

Figure 1: Solutions of equation (10) for $\kappa / \sigma^{2}=1,1 / 2$ (middle) and $1 / 4$.

Thus the magnification exponent depends only on the local input probability density $M \sim P^{\rho(P)}$, and we have $\rho_{q}=\frac{d Y}{d X}=\rho+X \frac{d \rho(X)}{d X}$, where $\rho=\rho_{q}$ for limiting cases with $d \rho(X) / d X \rightarrow 0$. For $\kappa \rightarrow 0$ the magnification exponent shifts from 1 to zero according to equation (10), rewritten as

$$
\frac{1}{\rho_{q}}=\frac{d X}{d Y}=\left(1+\frac{\kappa}{\sigma^{2}} e^{-Z}\right)=\left(1+\frac{\kappa}{\sigma^{2}} \frac{1}{\bar{P} M}\right) .
$$

Finally we remark that the decomposition (6) of the parallel update rule to update responses to the stimuli is not unique. Especially the elastic term can be decomposed in a siutable stimulus-dependent manner so that elasticity is appended only in vicinity of the stimulus. This Local Elastic Net reads

$$
\delta \mathbf{w}_{\mathbf{r}}=\eta \cdot\left\{A^{\sigma}\left(\mathbf{v}, \mathbf{w}_{\mathbf{r}}\right) \cdot\left(\mathbf{v}-\mathbf{w}_{\mathbf{r}}\right)+\kappa\left((1-\nu) \cdot A^{(\alpha \sigma)}\left(\mathbf{v}, \mathbf{w}_{\mathbf{r}}\right)+\nu\right) \cdot \Delta \mathbf{w}_{\mathbf{r}}\right\},
$$

where $A$ is a normalized gaussian function of distance, $\alpha \simeq 1$ and $0 \leq \nu \leq 1$. A small global elasticity (e.g. $\nu=0.05$ ) smoothes fluctuations, but the "forgetting" due to global relaxation is reduced which improves convergence. The Magnification law of the Local Elastic Net is similar as for the Elastic Net [9]. 


\section{Numerical Verification of the Magnification Law}

To calculate the asymptotic level density numerically, we considered the map of the unit interval to a onedimensional neural chain of 100 neurons with fixed first and last neuron. The learning rate was 0.5 . The stimulus probability density was chosen exponentially as $\exp (-\beta w)$ with $\beta=4$. After an adaptation process of $5 \cdot 10^{7}$ steps further $10 \%$ of learning steps were used to calculate average slope and its fluctuation (shown in brackets) of $\log J$ as a function of $\log P$. (The first and last $10 \%$ of neurons were excluded to eliminate boundary effects). The (local) magnification exponents were obtained as

\begin{tabular}{|l|c|c|c|c|}
\hline$\downarrow \sigma \quad \kappa \rightarrow$ & 0.24 & 0.024 & 0.0024 & 0.00024 \\
\hline 0.0003 & $0.00(0.01)$ & $0.03(0.01)$ & $0.15(0.02)$ & $0.29(0.03)$ \\
\hline 0.001 & $0.03(0.01)$ & $0.03(0.01)$ & $0.15(0.02)$ & $0.28(0.02)$ \\
\hline 0.003 & $0.04(0.01)$ & $0.03(0.01)$ & $0.23(0.01)$ & $0.49(0.01)$ \\
\hline 0.01 & $0.03(0.01)$ & $0.25(0.01)$ & $0.77(0.02)$ & $0.96(0.06)$ \\
\hline 0.03 & $0.23(0.01)$ & $0.70(0.03)$ & & \\
\hline
\end{tabular}

For the Elastic Net the parameter choice appeared crucial: Same as in the TSP application [7] the optimal choice of $\sigma$ as the average distance (in input space) between two adjacent neurons seems to be appropriate. For larger $\sigma$ clearly clustering phenomena appear due to the fact that too many neurons fall in the Gaussian neighborhood of the stimulus. For large $\kappa / \sigma^{2}$ the exponent decreases to zero, as given by the theory. For small $\kappa / \sigma^{2}$ the exponent first increases near to 1 but simultaneously instability due to clustering arises (last row).

Whereas the simulation validates the exact result, appropriate adjustment of $\kappa / \sigma^{2}$ between optimal mapping and stability remains difficult and becomes intractable for large-scale variations of the input probability density.

\section{References}

[1] T. Kohonen 1982. Biological Cybernetics 43, 59-69.

[2] K. Obermayer, G. G. Blasdel, and K. Schulten 1992. Phys. Rev. A 45, 7568-7589.

[3] H. Ritter, T. Martinetz, and K. Schulten 1992. Neural Computation and Self-Organizing Maps. Addison-Wesley.

[4] R. Durbin and D. Willshaw 1987. Nature 326, 689-691.

[5] P. D. Simic 1990. Network 1, 89-103.

[6] R. Durbin, R. Szeliski, and A. Yuille 1989. Neural Computation 1, 348-358.

[7] M. W. Simmen 1991. Neural Computation 3, 363-374.

[8] J. Hertz, A. Krogh, and R. G. Palmer 1991. Intr. to the Theory of Neural Comp. Addison-Wesley, Reading, MA.

[9] J. C. Claussen (born Gruel) 1992. Diploma thesis, Kiel, J. C. Claussen (born Gruel) and H. G. Schuster 1994. Preprint.

[10] T. Kohonen 1991. in: Artificial Neural Networks, ed. T. Kohonen et al. North-Holland, Amsterdam.

[11] R. Linsker 1989. Neural Computation 1, 402-411.

[12] H. Ritter and K. Schulten 1986. Biological Cybernetics 54, 99-106.

[13] D. R. Dersch and P. Tavan 1995. IEEE Trans. Neur. Netw. 6, 230-236.

[14] H. Ritter 1991. IEEE Transactions on Neural Networks 2, 173-175.

[15] P. D. Simic 1994. Private communication. 